\title{
Synthesis and Antibacterial Studies of Some Reduced Schiff base Derivatives
}

\author{
UDAYSINHA PATIL' ${ }^{1}$ ASIF KHAN ${ }^{1}$, AARTI NAGARSEKAR ${ }^{1}$, \\ MUSTAPHA MANDEWALE ${ }^{1}$ and RAMESH YAMGAR ${ }^{\star 2}$
}

\begin{abstract}
1Department of Chemistry, Government of Maharashtra's Ismail Yusuf College of Arts, Science and
Commerce, Jogeshwari (East), Mumbai, India.

${ }^{2}$ Department of Chemistry, Chikitsak Samuha's Patkar-Varde College of Arts, Science and

Commerce, Mumbai, India.
\end{abstract}

http://dx.doi.org/10.13005/ojc/340616

Received: July 13, 2018; Accepted: December 09, 2018)

\begin{abstract}
A series of $\mathrm{N}, \mathrm{N}$-substituted ethylene-1,2-diamine derivatives have been prepared from reaction of 2-hydroxybenzaldehyde derivatives and 1,2-diamine derivatives in the presence of $\mathrm{NaBH}_{4}$ through Schiff base intermediate. The synthesized compounds were screened for their antibacterial activities. Compound SB01, SB02 and SB09 displayed significant activity at MIC ranges from 0.40-6.25 $\mu \mathrm{g} / \mathrm{mL}$.
\end{abstract}

Keywords: Schiff bases, Reduction, Antibacterial, MIC, Antituberculosis, Bioactive.

\section{INTRODUCTION}

Schiff bases are synthesized typically by the condensation reaction of a primary amine and an aldehyde/ketone. The resultant compound, $\mathrm{R} 1 \mathrm{R} 2 \mathrm{C}=\mathrm{NR} 3$ is called a Schiff base, where $\mathrm{R} 1$ is an aryl or alkyl group, R2 is a hydrogen atom and R3 is either an alkyl or aryl group. The ary substituted Schiff bases are considerably more stable and easily prepared, while Schiff bases with alkyl substituents are comparatively less stable ${ }^{1}$, while those of aromatic aldehydes having effective conjugation are more stable. In general, aldehydes are more reactive than ketones in condensation reactions, leading to the formation of Schiff bases as the reaction center of aldehydes are sterically less hindered than that of ketone. Moreover, the extra carbon of ketone releases more electron density to the azomethine carbon and therefore makes the ketone less electrophilic as compared to aldehydes ${ }^{2}$.

Schiff base derivatives have a wide variety of applications in pharmaceutical, analytical chemistry $^{3-4}$. Transition metal complexes of Schiff bases have extremely significant comprising enormous areas of coordination compounds ${ }^{5}$. The synthesis of symmetrical Schiff bases obtained from reaction of carbonyl compounds, with substituted diamines in the ratio 1:2 and various aldehyde/ketone derivatives. Recently large amount of effort has been taken for the preparation and identification of different coordination complexes of Schiff base ligands ${ }^{6}$.

This is an Open Access article licensed under a Creative Commons license: Attribution 4.0 International (CC- BY). Published by Oriental Scientific Publishing Company @ 2018 
Schiff base ligands are important in the studies of coordination complexes since they essentially produce stable complexes ${ }^{7}$. Schiff base reactions are significant in organic reactions for preparing carbon-nitrogen bonds. Schiff bases have often known to form chelates type complexes. The suitabely positioned heteroatoms like $\mathrm{O}, \mathrm{N}$ and $\mathrm{S}$ are key atoms in the formation of coordination complexes in the structures of various metallobiomolecules ${ }^{8}$. This class of compounds have been widely studied for their antiviral, antifungal, anticancer, antibacterial and herbicidal activities ${ }^{9-11}$. It is common that the incorporation of metal ions to structure of biologically important compounds may improve their potencies.

The salophens exhibit flexible electronic, steric and lipophilic nature. These type of componds can be easily synthesized by the reaction of o-hydroxybenzaldehyde and 1,2-diamines. Salophen compounds with nitrogen and oxygen atoms are significant as their metal complexes show broad range of applications in a variety of reactions as homogeneous catalysis ${ }^{12-13}$, Oxidation ${ }^{14}$, hydroxylation ${ }^{15}$, Epoxidation ${ }^{16}$, Polymerization ${ }^{17}$, Hydrogenation ${ }^{18}$. These compounds also found applications as electroluminescent materials ${ }^{19-20}$, optical devices ${ }^{21}$, electrochemical sensors ${ }^{22}$, antifungal ${ }^{23}$ and antimicrobial activity ${ }^{24}$.

Generally Schiff bases have tendence to under go hydrolysis in solution phase. This problem overcome by reducing the imine bond by using common and mild reducing agent like Sodium borohydride. This could improve properties of the the reduced Schiff base and the resultant compound could be more flexible and non-planar while coordinating to a metal ion ${ }^{25-26}$.

Recently it is reported that reduced Schiff base derivative showed significant antimicrobial activity at low concentration level ${ }^{27}$. Voronova et al., ${ }^{28}$ reported excellent catalyst for the Sonogashira coupling reaction. Further, various types of reduced Schiff base ligands and their lorn centered complexes have gained mored attraction in the field of medicinal chemistry due to their effective DNA cleavage activity 29. It is evident from the literature that partially reduced Schiff base moieties are known to show excellent catalyst for oxidation of various phenols ${ }^{30}$.

Based on above observation and in extension of our research work for finding better antimicrobial copunds ${ }^{31-36}$ we found that it was worth trying to synthesize various reduced Schiff bases derivatives with expected antibacterial activity.

\section{EXPERIMENTAL}

\section{General procedure for preparation of schiff bases}

A solution of aldehyde derivative $(4 \mathrm{mmol})$ and 1, 2-diamine derivative 1a-1c $(2.0 \mathrm{mmol})$ in $\mathrm{EtOH}$ $(8 \mathrm{~mL})$ maintained for $2 \mathrm{~h}$ at $70^{\circ} \mathrm{C}$ under stirring. Reaction completion monitored with TLC. Reaction mass cooled to room temperature and the solid obtained was filtered and dried.

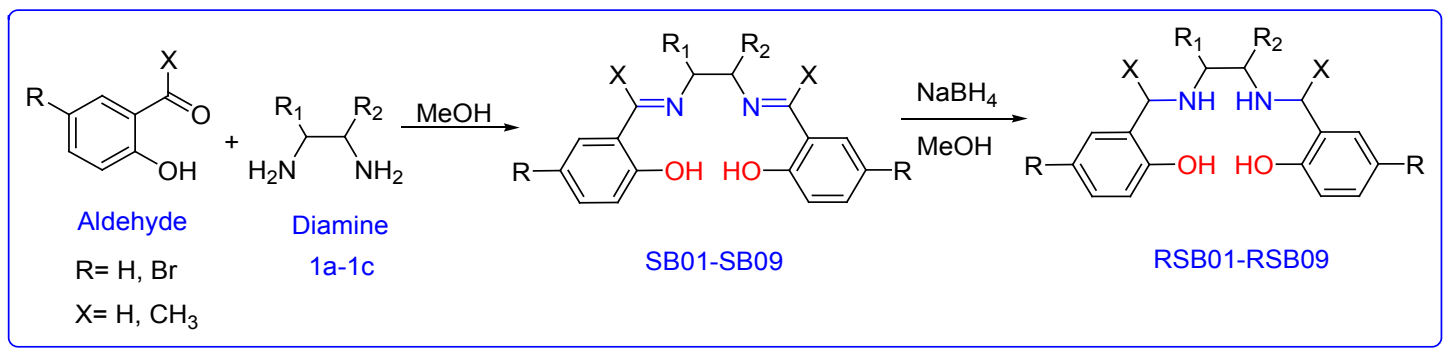

Fig. 1. Preparation of [RSB01-RSB09]

2,2'-\{ethane-1,2-diylbis[azanylylidene(E) methany Iylidene]\}diphenol [SB01]

m.p.: 125-1270 C; Colour:Yellow; EIMS [M+2]: 269.23; FTIR(KBr cm$\left.{ }^{-1}\right)$ : 3025, 2940, 2906, 2873, 2738, 1635, 1477, 1361, 1276, 1186, 1033, 827, 777, 628; ${ }^{1} \mathrm{H}-\mathrm{NMR}\left(300 \mathrm{MHz}\right.$, DMSO- $\left.{ }_{\mathrm{d} 6}\right) \delta: 3.895(\mathrm{~s}, 2 \mathrm{H})$, 6.825-6.884 (m, 4H), 7.271-7.409 (m, 4H), $8.565(\mathrm{~m}$,
4H) $13.358(\mathrm{~s}, 2 \mathrm{H})$; Elemental analysis $\left[\mathrm{C}_{16} \mathrm{H}_{16} \mathrm{~N}_{2} \mathrm{O}_{2}\right]$ : observed (Calculated): C 71.92\% (71.62\%), H 5.87\% (6.01\%), N 10.76\% (10.44\%).

\section{2,2'-\{1,2-Phenylenebis[azanylylidene(e)methany} Iylidene]\}diphenol [sb02]

m.p.: 154-156² C; Colour:Yellow; EIMS [M+2]: 318.22; 
$\mathrm{FTIR}\left(\mathrm{KBr} \mathrm{cm}{ }^{-1}\right): 3056,2734,1614,1562,1481$ 1363, 1276, 1191, 977, 910, 759; ' ${ }^{\mathrm{H}}$ NMR (300 $\mathrm{MHz}_{\text {, DMSO- }}$ ) $\delta: 6.930-6.978(\mathrm{~m}, 4 \mathrm{H}), 7.398-7.430$ $(\mathrm{m}, 4 \mathrm{H}), 7.635-7.658(\mathrm{~m}, 4 \mathrm{H}), 8.916(\mathrm{~s}, 2 \mathrm{H}), 12.904$ $(\mathrm{s}, 2 \mathrm{H}) ;{ }^{13} \mathrm{C}$ NMR $\left(75 \mathrm{MHz}, \mathrm{DMSO}_{\mathrm{d} 6}\right) \delta: 162.2,162.1$, 161.3, 161.1, 136.3, 136.2, 132.4, 132.3, 131.9, $131.7,129.2,129.1,124.3,124.2,119.3,119.2$, 119.1, 119.1, 117.3, 117.1; Elemental analysis $\left[\mathrm{C}_{20} \mathrm{H}_{16} \mathrm{~N}_{2} \mathrm{O}_{2}\right]$ : observed (Calculated): C $75.79 \%$ (75.93\%), H 5.16\% (5.10\%), N 8.94\% (8.86\%).

\section{2,2'-\{(4-methyl-1,2-phenylene)bis[azanylylidene} (E) methanylylidene]\} diphenol [SB03]

m.p.: $162-164^{\circ} \mathrm{C}$; Colour: Yellow; EIMS $[\mathrm{M}+\mathrm{H}]:$ 331.43; FTIR(KBr cm $\left.\mathrm{Cm}^{-1}\right): 3054,2985,2917$, 2713, 1616, 1563, 1486, 1365, 1278, 1189, 950, 757, 638; ${ }^{1} \mathrm{H}$ NMR (300 MHz, DMSO- $\left.{ }_{\mathrm{d} 6}\right) \delta: 2.389$ (s, 3H), 6.936-6.973 (m, 4H), 7.204-7.282 (m, 2H), 7.379-7.405 (m, 3H), 7.630-7.677 (m, 2H), 8.927 (s, 2H), 12.950 (s, 1H), $13.070(\mathrm{~s}, 1 \mathrm{H}) ;{ }^{13} \mathrm{C}$ NMR $\left(75 \mathrm{MHz}, \mathrm{DMSO}_{\mathrm{d}}\right) \delta: 162.3,162.1,161.2,161.1$, 136.3, 136.2, 132.4, 132.4, 132.3, 131.8, 131.7, $126.1,125.1,124.5,119.2,119.2,119.1,119.1$, 117.3, 117.1, 21.2; Elemental analysis $\left[\mathrm{C}_{21} \mathrm{H}_{18} \mathrm{~N}_{2} \mathrm{O}_{2}\right]$ : observed (Calculated): C 76.40\% (76.34\%), H 5.41\% (5.49\%), N 8.55\% (8.48\%).

\section{2-((E)-(2-((E)-5-bromo-2-hydroxybenzylideneamino) ethylimino)methyl)-4-bromophenol [SB04]}

m.p.: 192-194 $\mathrm{C}$; Colour: Yellow; EIMS [M+2]: 427.5; $\mathrm{FTIR}\left(\mathrm{KBr} \mathrm{cm}{ }^{-1}\right): 3026,2940,2906$, 2873, 2738, 1635, 1567, 1477, 1392, 1361, 1276, 1218, 1186, 1079, 1033, 977, 914, 827, 777, 692, 628; 'H-NMR (300 MHz, DMSO- ${ }_{\mathrm{d} 6}$ ) $\delta: 3.922$ (s, $2 \mathrm{H}), 6.823-6.853(\mathrm{~m}, 3 \mathrm{H}), 7.438-7.650(\mathrm{~m}, 3 \mathrm{H})$, $8.57(\mathrm{~m}, 4 \mathrm{H}) 13.45$ (s, 2H); Elemental analysis $\left[\mathrm{C}_{16} \mathrm{H}_{14} \mathrm{Br}_{2} \mathrm{~N}_{2} \mathrm{O}_{2}\right]$ : observed (Calculated): C $45.14 \%$ (45.1\%), H 3.38\% (3.31\%), N 6.60\% (6.67\%).

\section{2-((E)-(2-((E)-5-bromo-2-hydroxybenzylideneamino)} phenylimino)methyl)-4-bromophenol. [SB05]

m.p.: $186-188^{\circ} \mathrm{C}$; Colour: Yellow; EIMS [M+2]: 475.41; FTIR(KBr cm c $\left.^{-1}\right): 3056,2734,1614$, $1585,1562,1481,1450,1363,1277,1192,1151$, $1045,977,910,856,831,787,759,640,580$;
${ }^{1} \mathrm{H}$ NMR (300 MHz, DMSO- $\left.{ }_{\mathrm{d} 6}\right) \delta: 3.335$ (s,2H), 7.222$7.899(\mathrm{~m}, 3 \mathrm{H}), 8.116-8.145(\mathrm{~m}, 3 \mathrm{H}), 8.925-8.953$ (m, 4H), 10.805 (s, 2H);; ${ }^{13} \mathrm{C}$ NMR $\left(75 \mathrm{MHz}\right.$, DMSO-$\left._{\mathrm{d} 6}\right)$ $\delta: 162.2,162.1,161.3,161.1,136.3,136.2,132.4$, 132.3, 131.9, 131.7, 129.2, 129.1, 124.3, 124.2, 119.3, 119.2, 119.1, 119.1, 117.3, 117.1; Elemental analysis $\left[\mathrm{C}_{20} \mathrm{H}_{14} \mathrm{Br}_{2} \mathrm{~N}_{2} \mathrm{O}_{2}\right]$ : observed (Calculated): C $50.61 \%$ (50.66\%), H 3.05\% (2.98\%), N 5.88\% (5.91\%).

\section{2-((E)-(2-((E)-5-bromo-2-hydroxybenzylideneamino)} -4-methylphenylimino)methyl)-4-bromophenol [SB06] m.p.: $187-189^{\circ} \mathrm{C}$; Colour: Yellow; EIMS $[\mathrm{M}+\mathrm{H}]:$ 487.54; FTIR(KBr cm$\left.{ }^{-1}\right): 3055,2985,2918$, 2713, 1616, 1564, 1487, 1365, 1279, 1190, 1151, 1115, 1032, 995, 908, 839, 767, 738, 638, 503; ${ }^{1} \mathrm{H}-\mathrm{NMR}\left(300 \mathrm{MHz}, \mathrm{DMSO}_{\mathrm{d} 6}\right) \delta: 2.389$ (s, 3H), 6.918$6.962(\mathrm{~m}, 3 \mathrm{H}), 7.228-7.898(\mathrm{~m}, 3 \mathrm{H})$, 8.909-8.917 (m, $3 \mathrm{H}), 12.910(\mathrm{~s}, 1 \mathrm{H}), 13.091(\mathrm{~s}, 1 \mathrm{H})$; Elemental analysis $\left[\mathrm{C}_{21} \mathrm{H}_{16} \mathrm{Br}_{2} \mathrm{~N}_{2} \mathrm{O}_{2}\right]$ : observed (calculated): C $51.61 \%$ (51.67\%), H 3.33\% (3.30\%), N 5.68\% (5.74\%).

2-((E)-1-(2-((E)-1-(2-hydroxyphenyl)ethylideneamino) ethylimino)ethyl)phenol [SB07]

m.p.:190-192 ${ }^{\circ} \mathrm{C}$; Colour: Yellow; EIMS [M+2]: 296.32; FTIR(KBr cm$\left.{ }^{-1}\right): 3458,3242,3093$, 2902, 1662, 1604, 1574, 1475, 1382, 1337, 1288, 1182, 1146, 1072, 1039, 934, 808, 656, 576; ${ }^{1} \mathrm{H}-\mathrm{NMR}\left(300 \mathrm{MHz}, \mathrm{DMSO}_{\mathrm{d} 6}\right) \delta: 2.608$ (s, 3H), 2.481 (s, 3H), 3.895 (s, 4H), 6.829-6.884 (m, 4H), 7.271$7.409(\mathrm{~m}, 4 \mathrm{H}), 13.358(\mathrm{~s}, 2 \mathrm{H})$; Elemental analysis $\left[\mathrm{C}_{18} \mathrm{H}_{20} \mathrm{~N}_{2} \mathrm{O}_{2}\right]$ : observed (Calculated): C $72.70 \%$ (72.95\%), H 6.84\% (6.80\%), N 9.42\% (9.45\%).

2-((E)-1-(2-((E)-1-(2-hydroxy phenyl)ethylideneamino) phenylimino)ethyl)phenol [SB08]

m.p.: 194-196² C; Colour:Yellow; MS [M+2]: 345.34; FTIR(KBr cm-1): 3319, 3157, 2983, 2877 , 2829, 1660, 1612, 1551, 1479, 1394, 1321, 1290, 1184, 1070, 1004, 933, 816, 752, 682, 634, 586.; ${ }^{1} \mathrm{H}$ NMR $\left(300 \mathrm{MHz}, \mathrm{DMSO}_{\mathrm{d6}}\right) \delta: 2.316(\mathrm{~s}, 6 \mathrm{H}), 6.607-$ $6.963(\mathrm{~m}, 4 \mathrm{H}), 7.360-7.410(\mathrm{~m}, 4 \mathrm{H}), 7.735-7.761(\mathrm{~m}$, $4 \mathrm{H}), 15.025(\mathrm{~s}, 2 \mathrm{H})$; Elemental analysis $\left[\mathrm{C}_{22} \mathrm{H}_{20} \mathrm{~N}_{2} \mathrm{O}_{2}\right]$ observed (Calculated): C 76.79\% (76.73\%), H 5.88\% (5.85\%), N 8.18\% (8.13\%). 
Table 1: Structure of Schiff bases [SB01-SB09]

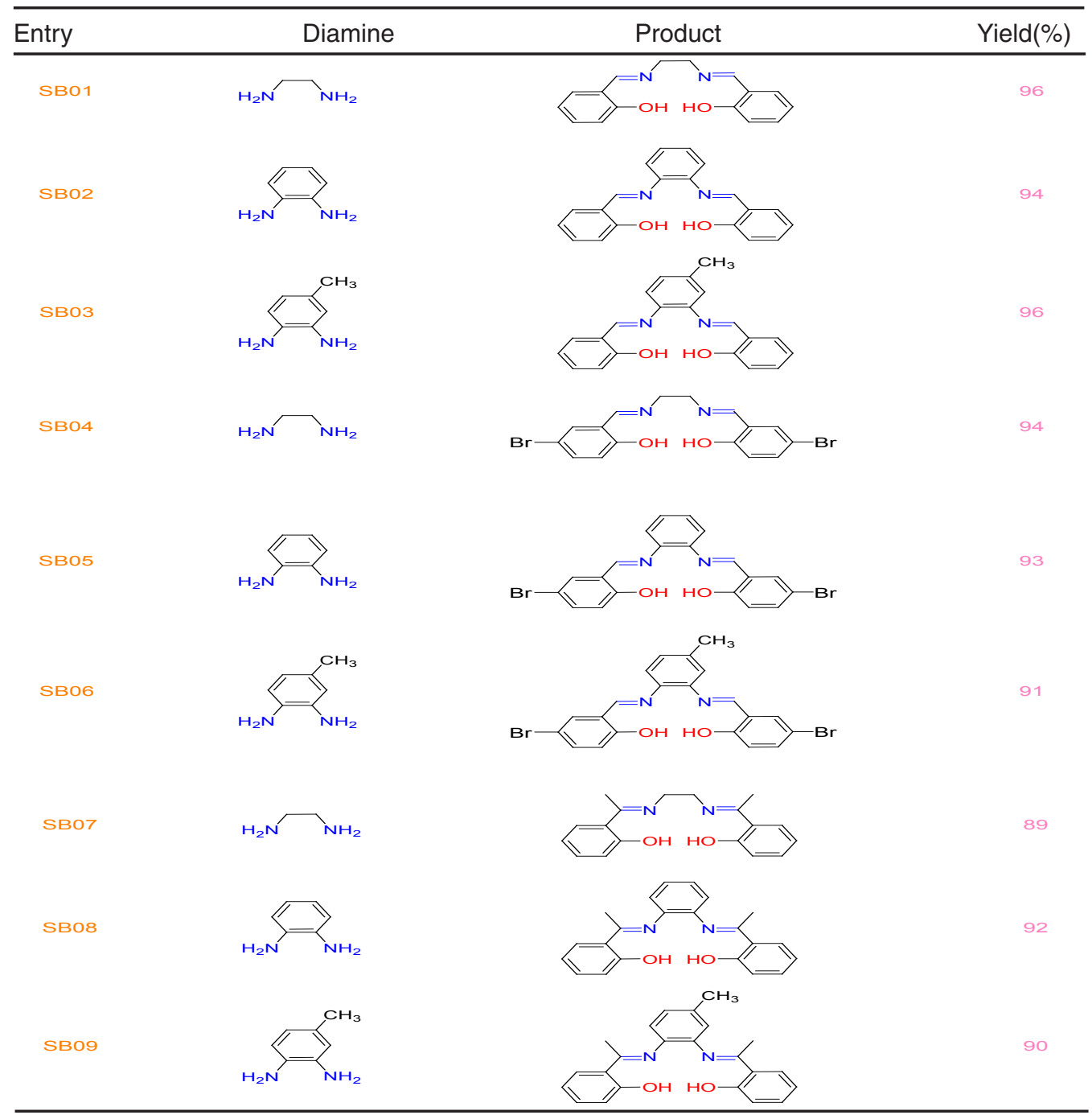

2-((E)-1-(2-((E)-1-(2-hydroxyphenyl)ethylideneamino)4-methylphenylimino)ethyl)phenol [SB09]

m.p.: 193-195 $\mathrm{C}$; Colour:Yellow; MS [M+H]:

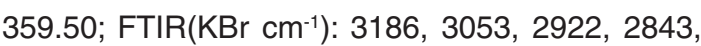
$1662,1616,1566,1479,1415,1255,1209,1178$, $1103,980,945,820,770,667,599,588 ;{ }^{1} \mathrm{H}$ NMR $(300$ $\left.\mathrm{MHz}, \mathrm{DMSO}_{\mathrm{d} 6}\right) \delta: 2.196(\mathrm{~s}, 6 \mathrm{H}), 2.389(\mathrm{~s}, 3 \mathrm{H}), 6.918-$ $6.962(\mathrm{~m}, 4 \mathrm{H}), 7.228-7.404(\mathrm{~m}, 4 \mathrm{H}), 7.539(\mathrm{~m}, 3 \mathrm{H})$, $12.910(\mathrm{~s}, 1 \mathrm{H}), 13.091(\mathrm{~s}, 1 \mathrm{H})$; Elemental analysis $\left[\mathrm{C}_{23} \mathrm{H}_{22} \mathrm{~N}_{2} \mathrm{O}_{2}\right]$ : observed (Calculated): $\mathrm{C} 77.40 \%$ (77.07\%), H 6.13\% (6.19\%), N 7.80\% (7.82\%).

\section{General procedure for preparation of reduced} schiff bases

The Schiff base $(2.27 \mathrm{mmol})$ dissolved in dichloromethane $(10 \mathrm{~mL})$. Reaction mass cooled at $0^{\circ} \mathrm{C}$ and 2-3 drops of conc. $\mathrm{KOH}$ solution added. The solution of $\mathrm{NaBH}_{4}(2.0 \mathrm{mmol})$ in methanol added dropwise. Reaction mass stirred for 4-5 $\mathrm{h}$ until the yellow colour disappeared. Reaction monitored on TLC. After completion of reaction solvent distilled and cold water $(10 \mathrm{~mL})$ added to dissolve residue. $\mathrm{pH}$ adjusted to $4-5$ by addition of dilute $\mathrm{HCl}$. The white solid filtered, washed with water dried.

\section{2,2'-[ethane-1,2-diylbis(azanediyl methylene)]diphenol [RSB01]}

m.p.: $118^{\circ} \mathrm{C}$; Colour: white; MS [M+2]: 273.99; $\mathrm{FTIR}\left(\mathrm{KBr} \mathrm{cm}^{-1}\right): 3490,3463,3363,3052$, 2942, 2852, 2593, 1602, 1479, 1417, 1353, 1268 
1189, 1126, 1074, 960, 815, 757, 626; ${ }^{1} \mathrm{H}$ NMR (300 $\left.\mathrm{MHz} \mathrm{DMSO}_{\mathrm{d} 6}\right) \delta: 2.481(\mathrm{~m}, 4 \mathrm{H}), 3.770(\mathrm{~s}, 4 \mathrm{H})$, $5.031(\mathrm{~s}, 2 \mathrm{H}), 6.658-6.686(\mathrm{~m}, 4 \mathrm{H}), 7.01-7.06(\mathrm{~m}$, $4 \mathrm{H}), 13.335(\mathrm{~s}, 2 \mathrm{H})$; Elemental analysis $\left[\mathrm{C}_{16} \mathrm{H}_{20} \mathrm{~N}_{2} \mathrm{O}_{2}\right]$ : observed (Calculated): C 70.63\% (70.56\%), H 7.31\% (7.40\%), N 10.34\% (10.29\%).

\section{2,2'-[1,2-phenylenebis(azanediylm ethylene)]diphenol [RSB02]}

m.p.: $138-140^{\circ} \mathrm{C}$; Colour: white; MS [M+]: 320.13; FTIR(KBr cm$\left.{ }^{-1}\right)$ : 3394, 3359, 3289, 3041, 2852, 1696, 1456, 1315, 1238, 1103, 1025, 929,750, 636; ' ${ }^{\mathrm{H}} \mathrm{NMR}\left(300 \mathrm{MHz}, \mathrm{DMSO}{ }_{\mathrm{d} 6}\right) \delta: 4.236(\mathrm{~s}, 4 \mathrm{H})$, $5.068(\mathrm{~s}, 2 \mathrm{H}), 6.37-6.450(\mathrm{~m}, 3 \mathrm{H}), 6.758-6.843(\mathrm{~m}$, $3 \mathrm{H})$, 7.023-7.070 (m, 3H), 7.176-7.222 (m, 3H), 10.805 (s, 2H); ${ }^{13} \mathrm{C} \mathrm{NMR}\left(75 \mathrm{MHz}\right.$, DMSO- $\left.{ }_{\mathrm{d} 6}\right) \delta: 157.6$, $157.5,141.1,141.0,131.8,131.8,128.9,128.8$, $123.2,123.1,122.6,122.6,121.8,121.8,120.6$, 120.6, 116.1, 116.0, 45.9, 45.8; Elemental analysis $\left[\mathrm{C}_{20} \mathrm{H}_{20} \mathrm{~N}_{2} \mathrm{O}_{2}\right]$ : observed (calculated): C 75.04\% (74.98\%), H 6.32\% (6.29\%), N 8.77\% (8.74\%).

\section{2,2'-[(4-methyl-1,2-phenylene) bis(azanediylmethylene)] diphenol [RSB03]}

m.p.: $136-138^{\circ} \mathrm{C}$; Colour: White; $\mathrm{MS}[\mathrm{M}+\mathrm{H}]$ : 335.57; $\operatorname{FTIR}\left(\mathrm{KBr} \mathrm{cm}^{-1}\right): 3390,3359,3278,2852$, 1696, 1455, 1305, 1251, 1110, 935, 844, 800, 752; ${ }^{1} \mathrm{H}$ NMR (300 MHz, DMSO- ${ }_{\mathrm{d} 6}$ ) $\delta: 2.055$ (s, 3H), 4.191 (s, 4H), 4.987 (s, 2H), 6.267-6.385 (m, 2H), 6.655-6.818 (m, 5H), $7.024(\mathrm{~m}, 2 \mathrm{H}), 7.172-7.195$ $(\mathrm{m}, 2 \mathrm{H}), 12.666(\mathrm{~s}, 1 \mathrm{H}), 12.904(\mathrm{~s}, 2 \mathrm{H}) ;{ }^{13} \mathrm{C}$ NMR $\left(75 \mathrm{MHz}, \mathrm{DMSO}_{\mathrm{d} 6}\right) \delta: 157.6,157.5,141.1,134.7$, $132.9,131.9,131.8,128.9,128.8,124.2,123.2$, $123.1,120.7,120.5,118.8,116.2,116.1,114.9$, 45.9, 45.8, 21.2, Elemental analysis $\left[\mathrm{C}_{21} \mathrm{H}_{22} \mathrm{~N}_{2} \mathrm{O}_{2}\right]$ : observed (Calculated): C 75.34\% (75.42\%), H6.72\% (6.63\%), N 8.30\% (8.38\%).

\section{2-((2-(5-bromo-2-hydroxybenzylamino)ethylamino)} methyl)-4-bromophenol [RSB04]

m.p.: $128-130^{\circ} \mathrm{C}$; Colour: white; MS [M+2] 273.99; FTIR(KBr cm$\left.{ }^{-1}\right)$ : 3303, 3232, 3097, 1621, 1579, 1502, 1481, 1384, 1309, 1282, 1267, 1224, 1195, 1130, 1079, 1022, 1000, 763, 734; ${ }^{1} \mathrm{H}$ NMR $(300 \mathrm{MHz}$,
DMSO- $\left._{\mathrm{d} 6}\right) \delta: 2.481(\mathrm{~m}, 2 \mathrm{H}), 2.608(\mathrm{~m}, 2 \mathrm{H}), 3.332(\mathrm{~s}$, $1 \mathrm{H}), 3.922(\mathrm{~s}, 1 \mathrm{H}), 5.031(\mathrm{~s}, 4 \mathrm{H}), 6.823-6.853(\mathrm{~m}, 3 \mathrm{H})$, 7.438-7.656(m, 3H), 13.450(s, 2H); Elemental analysis $\left[\mathrm{C}_{16} \mathrm{H}_{18} \mathrm{Br}_{2} \mathrm{~N}_{2} \mathrm{O}_{2}\right]$ : observed (Calculated): C $44.70 \%$ (44.68\%), H 4.25\% (4.22\%), N 6.48\% (6.51\%).

2-((2-(5-bromo-2-hydroxybenzyl amino)phenylamino) methyl)-4-bromophenol [RSB05]

m.p.: $132-134^{\circ} \mathrm{C}$; Colour: white; MS $[\mathrm{M}+]$ : 475.97; FTIR(KBr cm$\left.{ }^{-1}\right): 3415$, 3193, 3116, 3008, 2954, 1617, 1567, 1471, 1432, 1286, 1281, 1238 , 1214, 1103, 966, 794, 752, 609; ' $\mathrm{H}$ NMR (300 $\left.\mathrm{MHz} \mathrm{DMSO}_{\mathrm{d} 6}\right) \delta: 4.236(\mathrm{~s}, 1 \mathrm{H}), 3.33(\mathrm{~s}, 1 \mathrm{H}), 5.082$ $(\mathrm{s}, 4 \mathrm{H}), 6.39-6.769(\mathrm{~m}, 3 \mathrm{H}), 6.902-7.289(\mathrm{~m}, 3 \mathrm{H})$, 7.489-7.902 (m, 4H), $12.666(\mathrm{~s}, 2 \mathrm{H})$; Elemental analysis $\left[\mathrm{C}_{20} \mathrm{H}_{18} \mathrm{Br}_{2} \mathrm{~N}_{2} \mathrm{O}_{2}\right]$ : observed (calculated): C $50.28 \%$ (50.24\%), H 3.84\% (3.79\%), N 5.82\% (5.86\%).

2-((2-(5-bromo-2-hydroxybenzylamino)-4-methylpheny lamino)methyl)-4-br omophenol [RSB06]

m.p.: $127-129^{\circ} \mathrm{C}$; Colour: white; $\mathrm{MS}[\mathrm{M}+\mathrm{H}]$ : 335.57; FTIR(KBr cm$\left.{ }^{-1}\right): 3517,3455,3397,3189$, 3143, 3066, 2886, 2807, 1612, 1573, 1517, 1430, 1376, 1294, 1236, 1191, 1130, 1047, 968, 968, 935, 848, 784, 745, 572, 524, 485; ' ${ }^{\mathrm{H}-N M R}(300 \mathrm{MHz}$, DMSO- $\left._{d 6}\right) \delta: 2.316(\mathrm{~s}, 3 \mathrm{H}), 4.146(\mathrm{~s}, 2 \mathrm{H}), 5.102$ (s, 4H), 7.056-7.282 (m, 3H), 7.363-7.643 (m, 3H), 7.813-8.146 (m, 3H), $15.122(\mathrm{~s}, 1 \mathrm{H}), 15.649$ (s, $1 \mathrm{H})$; Elemental analysis $\left[\mathrm{C}_{21} \mathrm{H}_{20} \mathrm{Br}_{2} \mathrm{~N}_{2} \mathrm{O}_{2}\right]$ : observed (calculated): C 51.30\% (51.24\%), H 4.08\% (4.10\%), N 5.63\% (5.69\%).

\section{2-(1-(2-(1-(2-hydroxyphenyl)ethyl amino)ethylamino) ethyl)phenol [RSB07]}

m.p.: $121-123^{\circ} \mathrm{C}$; Colour: white; MS $[\mathrm{M}+1]$ : 301.23; FTIR(KBr cm$\left.{ }^{-1}\right): 3227,3153,3045$, 2998, 2944, 2892, 2840, 1617, 1556, 1488, 1432 , 1413, 1290, 1261, 1213, 1151, 1071, 956, 898, 846, 755, 684, 636, 603, 426; ' ${ }^{\mathrm{H}} \mathrm{NMR}(300 \mathrm{MHz}$, DMSO- $\left._{\mathrm{d} 6}\right) \delta: 1.288(\mathrm{~s}, 6 \mathrm{H}), 3.836(\mathrm{~m}, 4 \mathrm{H}), 3.857$ $(\mathrm{s}, 2 \mathrm{H}), 5.05(\mathrm{~s}, 2 \mathrm{H}), 6.360-6.723(\mathrm{~m}, 4 \mathrm{H}), 6.992$ $7.056(\mathrm{~m}, 4 \mathrm{H}), 12.259(\mathrm{~s}, 2 \mathrm{H})$; Elemental analysis $\left[\mathrm{C}_{18} \mathrm{H}_{24} \mathrm{~N}_{2} \mathrm{O}_{2}\right]$ : observed (Calculated): C $71.90 \%$ (71.97\%), H 8.10\% (8.05\%), N 9.39\% (9.33\%). 
Table 2: Structures of reduced Schiff bases [RSB01-RSB09]

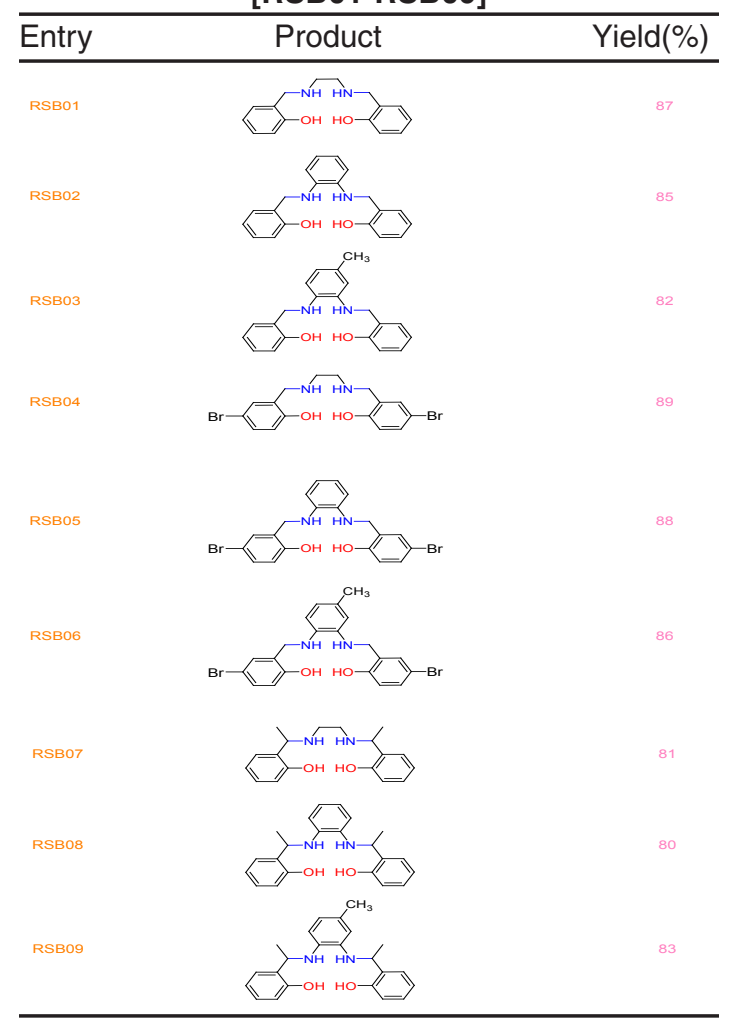

2-(1-(2-(1-(2-hydroxyphenyl)ethyl amino)phenylamino) ethyl)phenol [RSB08]

m.p.: $136-138^{\circ} \mathrm{C}$; Colour: white; $\mathrm{MS}[\mathrm{M}+]$ ]:348.78; FTIR(KBr cm-1): 3417, 3273, 3190, 1620, 1579, 1508, 1454, 1402, 1299, 1232, 1165, 1082, 1057, 1014, 879, $804,687,613,518 ;{ }^{\prime} \mathrm{HNMR}\left(300 \mathrm{MHz}, \mathrm{DMSO}_{-\mathrm{d}}\right) \delta: 2.045$ $(\mathrm{s}, 6 \mathrm{H}), 2.861(\mathrm{~s}, 2 \mathrm{H}), 5.214(\mathrm{~s}, 2 \mathrm{H}), 6.664-7.343(\mathrm{~m}, 4 \mathrm{H})$, 7.508-7.532 (m, 4H), 7.735-7.849 (m,4H), $12.031(\mathrm{~s}, 2 \mathrm{H})$; Elemental analysis $\left[\mathrm{C}_{22} \mathrm{H}_{24} \mathrm{~N}_{2} \mathrm{O}_{2}\right]$ : observed (Calculated): C $75.78 \%$ (75.83\%), H $6.90 \%$ (6.94\%), N $8.10 \%$ (8.04\%).

2-(1-(2-(1-(2-hydroxyphenyl) ethylamino)-4methylphenylamino) ethyl) phenol [RSB09]

m.p.: $122-124^{\circ} \mathrm{C}$; Colour: white; MS $[\mathrm{M}+\mathrm{H}]: 363.24 ; \mathrm{FTIR}\left(\mathrm{KBr} \mathrm{cm}^{-1}\right): 3539,3433,3118$, 3003, 2938, 2843, 1657, 1564, 1471, 1425, 1329, 1292, 1263, 1232, 1182, 1101, 976, 865, 738, 595; ${ }^{1} \mathrm{H}-\mathrm{NMR}\left(300 \mathrm{MHz}, \mathrm{DMSO}_{\mathrm{d} 6}\right) \delta: 2.371(\mathrm{~s}, 6 \mathrm{H})$, $2.555(\mathrm{~s}, 3 \mathrm{H}), 3.338(\mathrm{~s}, 2 \mathrm{H}), 5.107$ (s, 2H), 7.201$7.253(\mathrm{~m}, 4 \mathrm{H}), 7.331-7.508(\mathrm{~m}, 4 \mathrm{H}), 7.708(\mathrm{~m}, 3 \mathrm{H})$, $11.781(\mathrm{~s}, 1 \mathrm{H}), 12.388(\mathrm{~s}, 1 \mathrm{H})$; Elemental analysis $\left[\mathrm{C}_{23} \mathrm{H}_{26} \mathrm{~N}_{2} \mathrm{O}_{2}\right]$ : observed (calculated): $\mathrm{C} 76.32 \%$ (76.26\%), H 7.22\% (7.23\%), N 7.70\% (7.73\%).

\section{Biological evaluation Anti-mycobacterial activity}

The anti-microbial activities of the newly prepared compounds evaluated against $M$. tuberculosis ATCC No- 27294 by microplate Alamar Blue assay (MABA). A blue colour in the well was indicates bacterial growth whereas pink colour show growth of bacteria.

\section{RESULTS AND DISCUSSIONS}

\section{Chemistry}

The target compounds RSB01-RSB09 were prepared as outlined in Fig. 1. The required Schiff bases (SB01-SB09) were obtained by condensation reaction of requisite aldehydes and amines. The compounds (RSB01-RSB09) were prepared by reduction of imine bond of compounds with reducing agent like $\mathrm{NaBH}_{4}$ in quantitative yield. Elemental analysis and spectral data (FTIR, ${ }^{1} \mathrm{H}$ and ${ }^{13} \mathrm{C}-\mathrm{NMR}, \mathrm{MS}$ ) confirmed the structure of the synthesized products. The IR spectrum of Schiff bases SB01-SB09 showed strong absorption bands at $1614-1616 \mathrm{~cm}^{-1}$ and $3100-3400 \mathrm{~cm}^{-1}$ due to imine $(-\mathrm{HC}=\mathrm{N}-)$ function and hydroxyl $(-\mathrm{OH})$ group respectively, The ${ }^{1} \mathrm{H}-\mathrm{NMR}$ spectrum of Schiff bases SB01-SB09 revealed, in addition to expected aromatic signals, three singlets at $\delta 8.91$ and 12.95 $\mathrm{ppm}$ are assignable to the azomethine proton $(-\mathrm{CH}=\mathrm{N}-)$ and hydroxyl proton $(-\mathrm{OH})$, respectively. In addition, the ${ }^{13} \mathrm{C}$-NMR spectrum of Schiff bases SB01-SB03 displayed typical peaks at $\delta 161.0-161.5$ ppm assignable to imine carbon. Moreover the mass spectrum of Schiff bases revealed molecular ion peak confirming corresponding molecular weight of target compounds.

The FTIR spectra of reduced Schiff base RSB02 showed broad peak in the region $3289-3394 \mathrm{~cm}^{-1}$ due to amino (-NH-) and hydroxyl group, respectively. The ${ }^{1} \mathrm{H}-\mathrm{NMR}$ spectrum of reduced Schiff bases RSB02 showed, in addition to expected aromatic signals, three singlets at $\delta 5.03$ and $13.33 \mathrm{ppm}$ are assignable to the amino (-NH-) and hydroxyl proton $(-\mathrm{OH})$, respectively. The singlet at $\delta 4.2$ due to $-\mathrm{CH}_{2}$ - group confirms the formation of reduced Schiff bases. Additionally the mass spectrum of reduced Schiff base RSB02 revealed molecular ion peak at $\mathrm{m} / \mathrm{z} 320.13$ confirming it's molecular weight of target compound. Similarly other reduced Schiff bases have been characterized. 
Table 3: Antibacterial activity of the test samples in MIC $(\mu \mathrm{g} / \mathrm{mL})$

\begin{tabular}{ccccccc}
\hline Sr. No. & Entry & M. Tuberculosis & E. coli & P. aeruginosa & S. aureus & B. subtilis \\
\hline 01 & SB-01 & 12.50 & 12.50 & 3.12 & 50.00 & 6.25 \\
02 & SB-02 & 25.00 & 25.00 & 3.12 & 6.25 & 3.12 \\
03 & SB-03 & 50.00 & 25.00 & 12.50 & 12.50 & 6.25 \\
04 & SB-04 & 50.00 & 50.00 & 50.00 & 6.25 & 12.50 \\
05 & SB-05 & 50.00 & 50.00 & 25.00 & 6.25 & 50.00 \\
06 & SB-06 & 50.00 & 50.00 & 12.50 & 6.25 & 12.50 \\
07 & SB-07 & 25.00 & 25.00 & 25.00 & 1.60 & 25.00 \\
08 & SB-08 & 25.00 & 50.00 & 6.25 & 12.50 & 12.50 \\
09 & SB-09 & 25.00 & 12.50 & 3.12 & 0.40 & 12.50 \\
10 & RSB-01 & 12.50 & 12.50 & 25.00 & 12.50 & 3.12 \\
11 & RSB-02 & 50.00 & 25.00 & 6.25 & 1.60 & 25.00 \\
12 & RSB-03 & 50.00 & 50.00 & 25.00 & 12.50 & 25.00 \\
13 & RSB-04 & 50.00 & 50.00 & 50.00 & 12.50 & 12.50 \\
14 & RSB-05 & 50.00 & 50.00 & 25.00 & 6.25 & 25.00 \\
15 & RSB-06 & 50.00 & 50.00 & 25.00 & 3.12 & 12.50 \\
16 & RSB-07 & 50.00 & 50.00 & 25.00 & 3.12 & 12.50 \\
17 & RSB-08 & 50.00 & 50.00 & 25.00 & 25.00 & 25.00 \\
18 & RSB-09 & 50.00 & 25.00 & 12.50 & 0.40 & 12.50 \\
19 & Ciprofloxacin & 3.125 & 2.00 & $<4.00$ & 2.00 & 2.00 \\
20 & Pyrazinamide & 3.125 & - & - & - & - \\
21 & Streptomycin & 6.250 & - & - & - & - \\
\hline
\end{tabular}

\section{Anti-mycobacterial activity}

The anti-mycobacterial effects of the schiff base derivatives compounds SB01-SB09 and RSB01-RSB09 against Mycobacterium tuberculosis. The Ciprofloxacin (MIC $3.12 \mu \mathrm{g} / \mathrm{mL}$ ), Pyrazinamide (MIC $3.12 \mu \mathrm{g} / \mathrm{mL}$ ) and Streptomycin (MIC 6.25 $\mu \mathrm{g} / \mathrm{mL}$ ) were used as references to compare the potency of the synthesized compounds. As shown in table 3 compounds SB01 has unpredictable high anti-tuberculosis activity against Mycobacterium tuberculosis as its MIC value is $12.50 \mu \mathrm{g} / \mathrm{mL}$. This could be due to formation a specific complex with cell wall protein and ultimately interfering in cell wall synthesis of Mycobacterium tuberculosis during cell mitosis phase of multiplication. The presence of active pharmacophore present in the molecular structure of the compound, like imine double bond between carbon and nitrogen and well positioned hydroxyl group, these structural units restrict in the mechanism of cell multiplication and hence stop further growth of $M$. tuberculosis. All the samples under study are diplaying different activity because of the effective barrier of an outer cell wall membrane of $M$. tuberculosis for entry of external compounds.

This outcome indicates that suitably placed pharmacophores improved the penetration into the cell wall of the M. tuberculosis, which translated into good activity. In addition, these compounds interfere the respiration process of the cell and thereby stop the synthesis of proteins. If the synthesis of proteins is inhibited then formation bacterial cell wall is not possible which ultimately results in cell death and therefore restricts further growth and infection of the bacteria.

The results of anti-mycobacterial activity of all compounds SB01-SB09 and RSB01-RSB09 is shown in Table 3. The compound RSB01-RSB09 showed poor activity as compared to SB01-SB09 suggesting that the imine moiety was important to activity. Clearly, the presence of Schiff base moiety is crucial for anti-tubercular activity.

\section{Antibacterial activity}

Ciprofloxacin was used as references to evaluate the potency of the synthesized compounds against Escherichia coli (MIC 2.00 $\mu \mathrm{g} / \mathrm{mL}$ ), Pseudomonas aeruginosa (MIC $<4.00$ $\mu \mathrm{g} / \mathrm{mL}$ ), staphylococcus aureus (MIC $2.00 \mu \mathrm{g} / \mathrm{mL}$ ), bacillus subtilis (MIC $2.00 \mu \mathrm{g} / \mathrm{mL}$ ). The compounds SB01-SB09 showed excellent antibacterial antibacterial activity as compared to their reduced forms RSB01- 
RSB09. This also predicts the importance of the imine bond for the antibacterial activity.

\section{CONCLUSION}

In conclusion, the synthesis of 18 compounds were easily performed with good yields. All compounds were tested against $M$. tuberculosis, E. coli, P. aeruginosa, S. aureus, B. subtilis three of them (SB01, SB02 and SB09) exhibit considerable activity when compared with standard drugs like Ciprofloxacin, Pyrazinamide and Streptomycin.

\section{ACKNOWLEDGEMENT}

The authors thank Principal and Head Department of Chemistry, Government of Maharashtra, Ismail Yusuf Arts, Science and Commerce College for providing research and library facilities. The authors also thank Management and Principal of C. S.'s Patkar-Varde College, Goregaon (W), Mumbai for their constant encouragement and support. The authors also acknowledge the help of Dr. Kishore Bhat of Governmental Dental College, Belgaum, for anti-TB study.

\section{REFERENCES}

1. Hine, J.; Yeh, C.Y. Equilibrium in formation and conformational isomerization of imines derived from isobutyraldehyde and saturated aliphatic primary amines. J. Am. Chem. Soc., 1967, 89, 2669-2676.

2. Fessenden, R.J.; Fessenden, J.S. Organic Chemistry, Brooks/Cole Publishing Company, USA, 1998.

3. Cimerman, Z.; Miljanic S.; Galic, N. Schiff bases derived from aminopyridines as spectrofluorimetric analytical reagents. Croatica Chemica Acta., 2000, 73(1), 81-95.

4. Elmali, A.; Kabak, M.; Elerman, Y. Ketoenol tautomerism, conformations and structure of N-(2-hydroxy-5-methylphenyl), 2-hydroxybenzaldehydeimine. J. Mol. Struct., 2000, 477, 151-158.

5. Anacona, J.R.; Bastardo E.; Camus, J. Manganese (II) and palladium (II) complexes containing a new macrocyclic Schiff base ligand: antibacterial properties. J. Trans. Met. Chem., 1999, 24(4), 478-480.

6. Trujillo, A.; Sinbandhit, S.; Toupet, L.; Carrillo, D.; Manzu C.; Hamon, J.R. OrganometallicInorganic Conjugated Unsymmetrical SchiffBase Hybrids. Synthesis, Characterization, Electrochemistry and X-ray Crystal Structures of Functionalized Trinuclear Iron-NickelRuthenium Dipolar Chromophores. J. Inorg. Organomet. Polym., 2008, 18(1), 81-99.

7. Clarke, B.; Clarke, N.; Cunningham, D.; Higgins, T.; McArdle, P. Ni Cholchu and M.O'Gara, Transition-metal Schiff-base complexes as ligands in tin chemistry. Part 7. Reactions of organotin(IV) Lewis acids with $[\mathrm{M}(\mathrm{L})]_{2}\left[\mathrm{M}=\mathrm{Ni}, \mathrm{Cu}\right.$ and $\mathrm{Zn} ; \mathrm{H}_{2} \mathrm{~L}=\mathrm{N}, \mathrm{N}^{\prime}$-bis (3-methoxysalicylidene)benzene-1,3-diamine and its -1,4-diamine analog]. J. Organomet. Chem., 1998, 559, 55-64.

8. Brown, D.H.; Smith, W.E. Enzyme ChemistryImpact and Applications, Chapmann and Hall, London, 1990.

9. Singh, K.; Barwa M.S.; Tyagi, P. Synthesis and characterization of cobalt(II), nickel(II), copper(II) and zinc(II) complexes with Schiff base derived from 4-amino-3-mercapto6-methyl-5-oxo-1,2,4-triazine. Eur. J. Med. Chem., 2007, 42(3), 394-402.

10. Cozzi, P.G.; Metal-Salen Schiff base complexes in catalysis: practical aspects. Chem. Soc. Rev., 2004, 33, 410-421.

11. Chandra S.; Sangeetika, J. EPR and electronic spectral studies on copper(II) complexes of some N-O donor ligands. J. Ind. Chem. Soc., 2004, 81(3), 203-206.

12. Bolton, R.; Danks T.N.; Paul, J.M. Preparation and reduction of some camphor imines. Tetrahedron Letters., 1994, 35, 3411-3412.

13. Kazemi, F.; Reza, A.; Sarvestani, E. Practical reduction of imines by $\mathrm{NaBH} 4 /$ alumina under solvent-free conditions: An efficient route to secondary amine. Chinese Chemical Letters., 2008, 19, 1167-1170.

14. Uchida, T.; Katsuki, T. Cationic Co(III)(salen)catalyzed enantioselective Baeyer-Villiger oxidation of 3-arylcyclobutanones using hydrogen peroxide as a terminal oxidant. Tetrahedron Letters., 2001, 42, 6911-6914.

15. Ligtenbarg, A.G.J.; Beuken, E.K.; Meetsma, A.; Veldman, N.; Smeets, W.J.J.; Spek, A.L.; Feringa, B.L. New mono- and di-nuclear complexes of Pd (II), Pt (II) and Ni (II) of PNNP ligands with a 2,2'-biaryl bridging unit. Journal of Chemical Society Dalton Transactions., 1998, 263-270. 
16. McGarrigle, E.M.; Gilheany, D.G. Chromium and Manganese salen Promoted Epoxidation of Alkenes, Chemical Reviews., 2005, 105; 1563-1602.

17. Coe, B.J.; Harris, J.A.; Brunschwig, B.S.; Asselberghs, I.; Clays, K.; Garin, J.; Orduna, J. Three-dimensional nonlinear optical chromophores based on metal-to-ligand charge-transfer from ruthenium (II) or iron (II) centers. Journal of American Chemical Society., 2005, 127, 13399-13410.

18. Nishibayashi, Y.; Takei, I.; Vemara, S.; Hidai, M.; Extremely High Enantioselective Redox Reaction of Ketones and Alcohols Catalyzed by $\mathrm{RuCl}_{2}(\mathrm{PPh} 3)$ (oxazolinylferrocenylphosphine). Organometallics., 1999, 18, 2291-2293.

19. T. Yu, W. Su, W. Li, Z. Hong, R. Hua and B. Li. A schiff base zinc complex and its electroluminescent properties. Thin solid Films., 2007, 515, 4080-4084.

20. Y.Yi, X. Q.Wei, M.G. Xie and Z.Y. Lu. Synthesis and color-tunable fluorescence properties of schiff base zinc (II) complexes used as electroluminescent materials. Chinese Chemical Letters., 2004, 15, 525-528.

21. T. Verbiest, S. Houbrechts, M. Kauranen, K. Clays, A. Persoons. Second-order nonlinear optical materials: recent advances in chromophore design, Journal of Material Chemistry., 1997, 7, 2175-2189.

22. T. Shamspur, I. Sheikhshoaie and M.H. Mashhadizadeh. Flame atomic absorption spectroscopy (FAAS) determination of iron (III) after preconcentration on to modified analcime zeolite with 5-((4- nitrophenylazo)$\mathrm{N}-\left(2^{\prime}, 4^{\prime}\right.$-dimethoxyphenyl))salicylaldimine by column method. Journal of Analytical Atomic Spectrometry., 2005, 20, 476-478.

23. G.B. Bagihalli, P.G. Avaji, S.A. Patil and P.S. Badami. Synthesis, spectral characterization, in vitro antibacterial, antifungal and cytotoxic activities of $\mathrm{Co}(\mathrm{II}), \mathrm{Ni}(\mathrm{II})$ and $\mathrm{Cu}(\mathrm{II})$ complexes with 1,2,4-triazole Schiff bases. European Journal of Medicinal Chemistry., 2008, 43, 2639-2649.

24. E. Keskioglu, A.B. Gunduzalp, S. Cete, F. Hamurcu and B. Erk. Cr (III), Fe (III) and $\mathrm{Co}$ (III) complexes of tetradentate (ONNO) Schiff base ligands: Synthesis, characterization, properties and biological activity. Spectrochimica Acta Part A., 2008,
70, 634-640.

25. Cho, B.T.; Kang, S.K. Clean and Simple Chemoselective Reduction of Imines to Amines Using Boric Acid-Activated Sodium Borohydride under Solvent-Free Conditions. Synlett., 2004, 9, 1484-1488.

26. Cho, B.T.; Kang, S.K. Direct and indirect reductive amination of aldehydes and ketones with solid acid-activated sodium borohydride under solvent-free conditions. Tetrahedron., 2005, 61, 5725-5734.

27. Musiliyu, A.; Musa, M.; Omar, F.; Khan, Aspedon, A.; Cooperwood, J.S. Synthesis and Antimicrobial Activity of $\mathrm{N}, \mathrm{N}^{\prime}-\mathrm{Bis}(2-$ hydroxylbenzyl)-1,2-ethanediamine Derivatives. Lett Drug Des Discov., 2010 7(3), 165-170.

28. Voronova, K.; Homolya, L.; Udvardy, A.; Benyei, A.C.; Ferenc J. Pd-TetrahydrosalanType Complexes as Catalysts for Sonogashira Couplings in Water: Efficient Greening of the Procedure. Chem Sus Chem., 2014, DOI: 10.1002/cssc.201402147

29. Routier, S.; Vezin, H.; Lamour E.; Bailly, E. DNA cleavage by hydroxy-salicylideneethylendiamine-iron complexes, Nucleic Acids Research., 1999, 27(21), 4160-4166.

30. Adam, W.; Saha-Moeller, C.; Ganeshpure, P. A convevient synthesis of nickel (II) and cobalt (II) complexes of unsymmetrical salen-type ligands and their applications as catalysts for the oxidation of 2,6-dimethylphenol and 1,5dihydronaphthalene by molecular oxygen. Indian Journal of Chemistry., 2004, 43A, 56-62.

31. Mandewale M.C.; Kokate, S.; Thorat, B.R.; Sawant, S.S.; Yamgar, R.S. Zinc complexes of hydrazone derivatives bearing 3,4-dihydroquinolin-2(1H)-one nucleus as new anti-tubercular agents. Arabian Journal of Chemistry.m, 2016, doi: 10.1016/j. arabjc.2016.07.016.

32. Mandewale, M.C.; Thorat, B.R.; Nivid, Y.; Jadhav, R.; Nagarsekar, A.S.; Yamgar, R.S. Synthesis, structural studies and antituberculosis evaluation of new hydrazone derivatives of quinoline and their Zn (II) complexes. Journal of Saudi Chemical Society.m, 2016, doi: http://dx.doi. org/10.1016/j.jscs.2016.04. 
33. Mandewale, M.C.; Thorat, B.R.; Shelke, D.; Yamgar, R.S. Synthesis and biological evaluation of new hydrazone derivatives of quinoline and their $\mathrm{Cu}$ (II) and $\mathrm{Zn}$ (II) complexes against Mycobacterium Tuberculosis. Bioinorganic Chemistry and Applications., 2015, 2015, 14. doi: http:// dx.doi.org/10.1155/2015/153015.

34. Mandewale, M.C.; Thorat, B.R.; Yamgar, R.S. Synthesis and anti-mycobacterium study of some fluorine containing Schiff bases of quinoline and their metal complexes., 2015, 7(5), 207-215.

35. Yamgar R.S.; Nivid, Y.; Nalawade, S.;
Mandewale, M.C.; Atram, R.G.; Sawant, S.S. Novel zinc (II) complexes of heterocyclic ligands as antimicrobial agents: synthesis, characterisation, and antimicrobial studies.m, 2014, doi: http:// dx.doi.org/10.1155/2014/276598

36. Patil, U.C; Mandewale M.C.; Thorat B.R.; Nagarsekar A.S.; Yamgar R.S. Transition Metal Tetrahydro-Salophen Type Complexes: Synthesis, Characterization and Antitubercular Studies, Asian J. Research Chem., 2016, 9(9), 425-434. DOI: 10.5958/09744150.2016.00064.X. 